\title{
A note on adding and deleting edges in hierarchical log-linear models
}

\author{
David Edwards
}

Received: 6 July 2010 / Accepted: 6 October 2011 / Published online: 18 October 2011

(C) The Author(s) 2011. This article is published with open access at Springerlink.com

\begin{abstract}
The operations of edge addition and deletion for hierarchical log-linear models are defined, and polynomial-time algorithms for the operations are given.
\end{abstract}

Keywords Graphical log-linear model · Dual representation · Hypergraph transversal

\section{Introduction}

Hierarchical log-linear models are widely used for modeling multivariate discrete data. The pattern of conditional independence relations induced by such a model may be displayed as an undirected graph, the so-called dependence graph of the model. Graphical log-linear models comprise a subclass of the hierarchical models that have unique dependence graphs, whereas distinct hierarchical models may have the same graph.

It is useful to modify such models by adding and deleting edges. For graphical models it is clear how to define these operations, but for hierarchical models it is less obvious. This note examines these operations and gives polynomial-time algorithms for them.

\section{Properties}

Write the set of discrete variables (factors) as $\Delta=\{a, b, c, \ldots\}$. We consider two classes of models: $\mathscr{L}$, the class of hierarchical log-linear models with main effects

D. Edwards $(\varangle)$

Department of Molecular Biology and Genetics, Science and Technology,

Aarhus University, Aarhus, Denmark

e-mail: David.Edwards@agrsci.dk 
on $\Delta$, and $\mathscr{G}$, the class of graphical log-linear models with main effects on $\Delta$. Note that $\mathscr{G}$ is a subclass of $\mathscr{L}$. We can represent a model $m \in \mathscr{L}$ as a list of the form $m=\left[g_{1}, g_{2}, \ldots, g_{k}\right]$, where the sets $g_{i} \subseteq \Delta$ are termed generators and represent the maximal model terms not set to zero. For $h \subseteq \Delta$, we write $h \in m$ if $h$ represents a model term not set to zero, so that $h \in m$ if and only if $h \subseteq g_{i}$ for some generator $g_{i}$ of $m$. Note that for simplicity we only consider models that include all factors in $\Delta$, so that $\bigcup_{i=1 \ldots k} g_{i}=\Delta$.

It is sometimes useful to represent models in a dual form (Edwards and Havránek $1985)$ by specifying the minimal model terms that are set to zero. We write this as $[. .]^{-}$. So, for example, the model for no three factor interaction may be written as $[a b, b c, a c]$ in the standard form, or equivalently as $[a b c]^{-}$in the dual form. Note that since we do not consider models with main effects set to zero, all dual generators have cardinality greater than one.

Graphical models are characterized by the edges that are present (that is, the twofactor interactions that are not set to zero). An interaction involving three or more factors is present whenever all the corresponding two-factor terms are present. In contrast, in a hierarchical model that is not graphical, one or more higher-order interaction terms are set to zero even though all corresponding two-factor interactions are not. For example, $[a b c, b d]=[a d, c d]^{-}$is graphical and $[a b, b c, a c, b d]=[a b c, a d, c d]^{-}$ is not.

Note that if a dual generator has cardinality three or more, this indicates that a higher-order interaction is set to zero even though all the corresponding two-factor terms are present. Thus $m \in \mathscr{G}$ if and only if all its dual generators have cardinality two. In other words, the dual representation of a graphical log-linear model is simply a list of missing edges. So for models in $\mathscr{G}$, the deletion or addition of an edge $e$ can be implemented by adding $e$ to or deleting $e$ from the dual representation.

As shown by Havránek (1982) (see also Edwards and Havránek 1985, Sect. 2), $\mathscr{L}$ forms a distributive lattice when it is endowed with meet and join operators. For two models $m_{1}$ and $m_{2}$ in $\mathscr{L}$, the join $m_{1} \vee m_{2}$ is the minimal supramodel of $m_{1}$ and $m_{2}$, and the meet $m_{1} \wedge m_{2}$ is the maximal submodel of $m_{1}$ and $m_{2}$. The join operation may be performed by concatenating generator lists

$$
\left[a_{1}, \ldots, a_{j}\right] \vee\left[b_{1}, \ldots b_{k}\right]=\left[\max \left(a_{1}, \ldots, a_{j}, b_{1}, \ldots b_{k}\right)\right]
$$

where $\max ()$ returns the maximal sets in the input list, or by forming all pairwise unions of dual generators, one from each list

$$
\left[a_{1}, \ldots, a_{j}\right]^{-} \vee\left[b_{1}, \ldots b_{k}\right]^{-}=\left[\min \left(a_{1} \cup b_{1}, \ldots, a_{j} \cup b_{k}\right)\right]^{-}
$$

where $\min ()$ returns the minimal sets in the input list.

Similarly, the meet operation may be performed by concatenating dual generator lists

$$
\left[a_{1}, \ldots, a_{j}\right]^{-} \wedge\left[b_{1}, \ldots b_{k}\right]^{-}=\left[\min \left(a_{1}, \ldots, a_{j}, b_{1}, \ldots b_{k}\right)\right]^{-}
$$


or by forming all pairwise intersections of generators, one from each list

$$
\left[a_{1}, \ldots, a_{j}\right] \wedge\left[b_{1}, \ldots b_{k}\right]=\left[\max \left(a_{1} \cap b_{1}, \ldots, a_{j} \cap b_{k}\right)\right]
$$

We now consider add and delete edge operations for models in $\mathscr{L}$. The natural way to extend the operations described above from $\mathscr{G}$ to $\mathscr{L}$ is to use the same definition, i.e., we delete (add) an edge $e$ to $m$ by adding $e$ to (deleting $e$ from) the dual representation of $m$.

For example, if $m=[a b, a c, b c, b d, c d]=[a b c, b c d, a d]^{-}$then adding $e=(a d)$ gives $m+e=[a b c, b c d]^{-}=[b c, a c d, a b d]$.

Similarly if $m=[a b c, b d, c d]=[b c d, a d]^{-}$then adding $e=(a d)$ gives $m+e=$ $[b c d]^{-}=[a c d, a b d, a b c]$.

Note that edge deletion may require the removal of redundant sets from the dual representation: for example, for $\Delta=\{a, b, c\}$, if $m=[a b, b c, a c]=[a b c]^{-}$, deleting $e=(a b)$ gives $m-e=[a b c, a b]^{-}=[a b]^{-}=[b c, a c]$.

The operations are simply characterized in the following lemma.

Lemma 1 As defined above, $m-e=m \wedge[e]^{-}$and $m+e$ is the maximal model $w$ for which $w-e=m$.

Proof That $m-e=m \wedge[e]^{-}$follows immediately from (1). Let $m$ be a model not containing $e$ and write $m=\left[g_{1}, \ldots g_{k}, e\right]^{-}$. Let $w$ be a maximal model satisfying $w-e=m$, and suppose $w=\left[a_{1}, \ldots a_{s}, b_{1}, \ldots b_{t}\right]^{-}$where $e \nsubseteq a_{i}$ for $i=1 \ldots s$ and $e \subseteq b_{i}$ for $i=1 \ldots t$. Then $m=w-e=\left[a_{1} \ldots a_{s}, e\right]^{-}$so $\left\{a_{1} \ldots a_{s}\right\}=\left\{g_{1} \ldots g_{k}\right\}$. Since $w$ is maximal $b_{1}=\ldots=b_{t}=\emptyset$ so $w=m+e$ is the unique maximal model for which $w-e=m$.

Alternatively, the add edge operation could be defined by setting $m+e$ to be the minimal model in $\mathscr{L}$ containing $m$ as a submodel and containing the edge $e$. This could simply be obtained by concatenating $e$ to the standard representation. However this would not coincide with the natural add edge operation for models in $\mathscr{G}$, and so would not serve our purpose.

A desirable property is commutativity. By the previous lemma, when $e$ is not present in $m,(m+e)-e=m$ holds. It would also be desirable that when $e$ is present in $m$, $(m-e)+e=m$ holds. We now find necessary and sufficient conditions for this.

To do this we must first define the local graphical property. We say that a model $m \in$ $\mathscr{L}$ containing an edge $e$ is locally graphical w.r.t. $e$ if no higher-order interaction term containing $e$ is set to zero when all corresponding lower-order interactions are present. That is to say, $m$ is locally graphical w.r.t. $e$ if $\forall f \supset e, e^{\prime} \in m \forall e^{\prime} \subset f \Rightarrow f \in m$. For example, $m=[a b c, b d, c d]=[b c d, a d]^{-}$is locally graphical with respect to $(a b)$ but not $(b c)$.

Lemma 2 A model $m \in \mathscr{L}$ containing $e$ is locally graphical with respect to $e$ if and only if no dual generator of $m$ contains $e$.

Proof Suppose first that $m$ is locally graphical with respect to $e$ but contains a dual generator $g$ such that $e \subseteq g$. Since $m$ contains $e, e \neq g$, so $g$ has cardinality three or 
more. Since $g$ is minimal, no lower-order interactions corresponding to subsets of $g$ are set to zero, contradicting the local graphical property.

For the converse, suppose that no dual generators of $m$ contain $e$ but that $m$ is not locally graphical with respect to $e$. Then there exists a set $f \notin m$ with $f \supset e$ and $e^{\prime} \in m \forall e^{\prime} \subset f$. Thus there exists a dual generator $g$ with $g \subseteq f$. Since $e^{\prime} \in m \forall e^{\prime} \subset f$, $g=f$. But then $g$ contains $e$, contrary to assumption.

Lemma $3(m-e)+e=m$ holds if and only if $m$ is locally graphical with respect to $e$.

Proof If $m$ is locally graphical with respect to $e$ then we can write it as $m=$ $\left[g_{1}, \ldots, \ldots g_{k}\right]^{-}$with $e \nsubseteq g_{i}$ for $i=1, \ldots, k$. Thus $m-e=\left[g_{1}, \ldots g_{k}, e\right]^{-}$ and so $(m-e)+e=\left[g_{1}, \ldots g_{k}\right]^{-}=m$, as required. Conversely, suppose $m$ is not locally graphical with respect to $e$. Then we can write $m=\left[g_{1}, \ldots, g_{j}, \ldots g_{k}\right]^{-}$ where $e \subset g_{i}$ for $i=1, \ldots, j$, for some $1 \leq j \leq k$. So $m-e=\left[g_{j+1}, \ldots g_{k}, e\right]^{-}$ and $(m-e)+e=\left[g_{j+1}, \ldots g_{k}\right]^{-} \neq m$, as required.

For example, consider $m=[a b c, b d, c d]=[b c d, a d]^{-}$once more. When $e=$ $(a b), m-e=[b c d, a d, a b]^{-}$and so $(m-e)+e=[b c d, a d]^{-}=m$, but when $e=(b c), m-e=[a d, b c]^{-}$and $(m-e)+e=[a d]^{-}=[a b c, b c d] \neq m$.

\section{Algorithms}

Although the edge addition and deletion operations described above are simply described and performed using the dual representation, this approach would be inefficient in practice. Common tasks such as computation of maximum likelihood estimates are closely tied to the standard model representation, and so it would be necessary to convert between the two representations. Algorithms to do this have been given by Edwards and Havránek $(1985,1987)$ but are inefficient for high dimensions. The problem has been well-studied in the computer science literature, where it is called hypergraph transversal: in general the running time is exponential in the number of generators in the input list (Eiter and Gottlob 1995).

Fortunately, however, more efficient algorithms for the edge operations based on the standard representation are available; these were given in Murphy and Rohl (1989). To delete $e=(u v)$ from $m=\left[g_{1}, \ldots, \ldots g_{k}\right]$, we compute

$$
m \wedge[e]^{-}=\left[g_{1}, \ldots, \ldots g_{k}\right] \wedge[\Delta \backslash\{u\}, \Delta \backslash\{v\}]
$$

using (2). This replaces all generators containing $e$ in $m$, i.e. of the form $e \cup s$ for $s \subset \Delta$, by $\{u\} \cup s$ and $\{v\} \cup s$ (and then redundant generators are removed).

To add an edge $e=(u v)$ to a model $m$, we need to find the maximal sets $s$ such that $\{u\} \cup s$ and $\{v\} \cup s$ are present in $m$, and then add generators of the form $e \cup s$ for these $s$. Clearly the resulting model, $m^{*}$ say, satisfies $m^{*}-e=m$. To see that it is maximal in this respect let $g$ be a generator of $m+e$. If $e \subseteq g$, then $\{u\} \cup s \in m$ and $\{v\} \cup s \in m$ for $s=g \backslash e$; otherwise $g \in m$. Thus all generators in $m+e$ not in $m$ must be of the form stated. 
To find the maximal sets we note that if there exist generators $g_{i}$ and $g_{j}$ such that $\{u\} \cup s \subseteq g_{i}$ and $\{v\} \cup s \subseteq g_{j}$, then $s \subseteq g_{i} \cap g_{j}$. Conversely, if $s=g_{i} \cap g_{j}$ where $u \in g_{i}$ and $v \in g_{j}$, then $\{u\} \cup s \subseteq g_{i}$ and $\{v\} \cup s \subseteq g_{j}$. It follows that

$$
\begin{aligned}
& \max \left\{s: \exists i, j \text { such that }\{u\} \cup s \subseteq g_{i},\{v\} \cup s \subseteq g_{j}\right\} \\
& \quad=\max \left\{g_{i} \cap g_{j}: u \in g_{i}, v \in g_{j}\right\} .
\end{aligned}
$$

So the following algorithm can be used to add the edge $e=(u v)$ to $m=\left[g_{1}, \ldots, g_{k}\right]$ :

for $i \leftarrow 1$ to $k$ do if $u \in g_{i}$

for $j \leftarrow 1$ to $k$ do if $v \in g_{j}$ add $\{u, v\} \cup\left(g_{i} \cap g_{j}\right)$ to $m$;

end for

end for

$m \leftarrow \max (m)$

Both algorithms involve a number of set operations that is quadratic in $k$, the number of input generators. Set operations can be implemented to run in linear time in terms of the number of set elements (see for example Golumbic 2004, App. B). Hence both algorithms run in polynomial time.

Acknowledgments Thanks are due to a reviewer whose comments improved the paper considerably.

Open Access This article is distributed under the terms of the Creative Commons Attribution Noncommercial License which permits any noncommercial use, distribution, and reproduction in any medium, provided the original author(s) and source are credited.

\section{References}

Edwards D, Havránek T (1985) A fast procedure for model search in multidimensional contingency tables. Biometrika 72:339-351

Edwards D, Havránek T (1987) A fast model selection for large families of models. J Am Stat Assoc 82:205-213

Eiter T, Gottlob G (1995) Identifying the minimal transversals of a hypergraph and related problems. Siam J Comput Sci Eng 24:1278-1304

Golumbic M (2004) Algorithmic graph theory and perfect graphs (Annals of discrete mathematics vol 57). North-Holland Publishing Co., Amsterdam

Havránek T (1982) Some complexity considerations concerning hypotheses in multidimensional contingency tables. In: Kozesnik J (ed) Transactions of the ninth Prague conference on information theory, statistical decision functions, random processes. Academic Press, Prague, pp 281-286

Murphy B, Rohl J (1989) Algorithm AS247: updating the sufficient configurations for fitting ZPA models to multidimensional contingency tables. J R Stat Soc Ser C 38:412-420 